\title{
1 Peptidoglycan associated cyclic lipopeptide disrupts viral infectivity
}

Bryan A. Johnson ${ }^{1}$, Adam Hage ${ }^{1}$, Birte Kalveram ${ }^{2}$, Megan Mears $^{2}$, Jessica A. Plante ${ }^{3,1}$, Sergio E. Rodriguez ${ }^{1}$, Zhixia Ding ${ }^{2,5}$, Xuemei Luo ${ }^{4,6}$, Dennis Bente ${ }^{1}$, Shelton S. Bradrick ${ }^{4}$, Alexander N. Freiberg ${ }^{2}$, Vsevolod Popov ${ }^{2,5}$, Ricardo Rajsbaum ${ }^{1,7}$, Shannan Rossi ${ }^{1,2}$, William K. Russell ${ }^{4,6}$, Vineet D. Menachery ${ }^{1,7}$;

$8{ }^{1}$ Department of Microbiology and Immunology, ${ }^{2}$ Department of Pathology, ${ }^{3}$ World Reference 9 Center for Emerging Viruses and Arboviruses, ${ }^{4}$ Department of Biochemistry and Molecular 10 Biology, ${ }^{5}$ UTMB Electron Microscopy Laboratory, ${ }^{6}$ UTMB Mass Spectrometry Facility, ${ }^{7}$ Institute for Human Infections and Immunity, University of Texas Medical Branch at Galveston 


\section{Abstract}

2 Enteric viruses exploit bacterial components including lipopolysaccharides (LPS) and

3 peptidoglycan (PG) to facilitate infection in humans. With origins in the bat enteric system, we

4 wondered if severe acute respiratory syndrome-coronavirus (SARS-CoV) or Middle East

5 respiratory syndrome-CoV (MERS-CoV) also use bacterial components to modulate infectivity.

6 To test this question, we incubated CoVs with LPS and PG and evaluated infectivity finding no

7 change following LPS treatment. However, PG from B. subtilis reduced infection $>10,000$-fold

8 while PG from other bacterial species failed to recapitulate this. Treatment with an alcohol

9 solvent transferred inhibitory activity to the wash and mass spectrometry revealed surfactin, a

10 cyclic lipopeptide antibiotic, as the inhibitory compound. This antibiotic had robust dose- and

11 temperature-dependent inhibition of $\mathrm{CoV}$ infectivity. Mechanistic studies indicated that surfactin

12 disrupts CoV virion integrity and surfactin treatment of the virus inoculum ablated infection in

13 vivo. Finally, similar cyclic lipopeptides had no effect on CoV infectivity and the inhibitory effect

14 of surfactin extended broadly to enveloped viruses including influenza, Ebola, Zika, Nipah,

15 Chikungunya, Una, Mayaro, Dugbe, and Crimean-Congo hemorrhagic fever viruses. Overall,

16 our results indicate that peptidoglycan-associated surfactin has broad virucidal activity and

17 suggest bacteria byproducts may negatively modulate virus infection. 


\section{Importance}

2 In this manuscript, we considered a role for bacteria in shaping coronavirus infection. Taking

3 cues from studies of enteric viruses, we initially investigated how bacterial surface components

4 might improve CoV infection. Instead, we found that peptidoglycan-associated surfactin is a

5 potent viricidal compound that disrupts virion integrity with broad activity against enveloped

6 viruses. Our results indicate that interactions with commensal bacterial may improve or disrupt

7 viral infections highlighting the importance of understanding these microbial interactions and

8 their implications for viral pathogenesis and treatment. 


\section{Introduction}

3 Commensal bacteria inhabit nearly every surface of the human body, influencing numerous host

4 processes $(1,2)$. While considered to serve a protective role, recent studies indicate enteric

5 viruses exploit bacterial envelope components to facilitate infection (3). Poliovirus was found to

6 bind both lipopolysaccharides (LPS) and peptidoglycan (PG) to enhance its thermostability and

7 receptor affinity, facilitating in vivo infection (3). Antibiotic depletion of commensal bacteria

8 inhibited oral poliovirus infection, but was rescued by recolonization, pretreatment of virus with

9 LPS, or bypassing the enteric system through intraperitoneal injection (3). Other viruses

10 including reovirus, mouse mammary tumor virus, and murine norovirus have been shown to use

11 similar mechanisms to facilitate infection $(3,4)$. Together, these results indicate a key role for

12 commensal bacteria in improving infectivity and pathogenesis of enteric viruses.

13 Like the enteric system, the respiratory tract harbors high levels of commensal bacteria (1).

14 Given the origins of Severe Acute Respiratory Syndrome-Coronavirus (SARS-CoV) and Middle

15 East Respiratory Syndrome (MERS)-CoV in the bat enteric system (5), we wondered if CoVs utilized bacterial components to facilitate infection. Previous work had identified a key role for

17 the TLR pathways in immunity to SARS-CoV with the absence of LPS binding TLR4 or its downstream adaptors resulting in augmented disease (6-8). Given the interactions observed between enteric viruses and bacterial components, CoVs may also use similar microbial components to improve infectivity and subsequently stimulate the TLR4 response.

21 In this study, we explored the relationship between bacterial surface components and CoV

22 infection. Surprisingly, we found PG from Bacillus subtilis reduced CoV infectivity. Using mass

23 spectrometry, we identified a cyclic lipopeptide (CLP), surfactin, as the molecule responsible for

24 CoV inhibition. Surfactin's inhibitory effect was dose and temperature dependent with treatment 
1 disrupting the integrity of the CoV particle. Notably, surfactin treatment of the inoculum ablated

2 CoV infection in vivo, but prophylactic treatment had no effect. Other similar CLPs had no effect

3 on CoV infectivity suggesting surfactin's virucidal properties were unique. Importantly, surfactin

4 treatment reduced the infectivity of several other enveloped viruses, including influenza A, Zika,

5 Dugbe, Nipah, Crimean-Congo Hemorrhagic Fever, chikungunya, Mayaro, Una, and Ebola

6 viruses. Together, these results demonstrate the efficacy of surfactin as a virucidal compound

7 and highlight the potential for microbial environment to modulate virus infection. 


\section{Results}

2 Peptidoglycan derived from $B$. subtilis reduces with coronavirus infectivity.

3 Given their origins in bat enteric systems, we wondered if CoVs might be stabilized by bacterial

4 components (5). To test this possibility, Human CoV-229E, a common cold associated CoV,

5 and MERS-CoV were treated with control (PBS), LPS (Escherichia coli) or PG (Bacillus subtilis)

6 and viral infectivity was determined (Fig. 1A). In contrast to enteric viruses, LPS had no effect

7 on CoV infectivity; however, the presence of PG from B. subtilis dramatically reduced the

8 infectivity of both HCoV-229E and MERS-CoV (Fig. 1B). The structure of PG varies

9 considerably between bacterial species (9), suggesting that PG from different bacteria may

10 have distinct effects on CoV infectivity. To explore this, we tested a diverse set of bacterial

11 derived PGs for the ability to modulate CoV infection (Fig. 1C). Notably, only PG derived from

12 B. subtilis reduced HCoV-229E and MERS-CoV infection, suggesting that interference with CoV

13 infectivity is not shared by PG from all bacterial species.

14 Next, we wondered if incubation temperature also played a role in $B$. subtilis PG reduction of

15 CoV infectivity. To investigate, HCoV-229E and MERS-CoV stocks were treated with B. subtilis

$\mathrm{PG}$ at room temperature $(\mathrm{RT}), 32^{\circ} \mathrm{C}$, or $37^{\circ} \mathrm{C}$ (Fig. 1 D-E). Interestingly, $\mathrm{PG}$ disruption of viral

17 infectivity was reduced at lower temperatures. For HCoV-229E, infectivity had a step-wise reduction with increasing temperature (Fig. 1D). In contrast, PG reduction of MERS-CoV infectivity was ablated at lower temperatures, with no significant loss of viral infectivity at either $\mathrm{RT}$ or $32^{\circ} \mathrm{C}$ (Fig. 1E). Together, these data indicate that the inhibitory effect of $B$. subtilis PG is

21 influenced by incubation temperature.

\section{Infectivity inhibition can be disassociated from PG}

23 Two possible scenarios explain why only B. subtilis PG reduces CoV infectivity: 1) B. subtilis PG 24 reduces infectivity directly, using unique structural features absent in PG from other bacteria; or 25 2) the $P G$ preparation contains another compound that mediates inhibition. To differentiate 26 these possibilities, we exploited the poor solubility of PG, washing it in a variety of solvents to 
separate its inhibitory effect (Fig. 2A). After three washes in PBS, PG maintained its reduction of HCoV-229E infectivity (Fig. 2A). In contrast, PG washed with either 100\% ethanol or DMSO lost the ability to inhibit HCoV-229E infectivity (Fig. 2A). These results suggest that the washes either modified the inhibitory capacity of PG or removed a soluble compound responsible for reducing CoV infectivity. To explore this, the supernatants from clarified PG samples were incubated with HCoV-229E (Fig. 2B). While the PBS, PBS control, and ethanol control had no inhibitory effect, the ethanol supernatant from PG potently reduced viral infectivity of HCoV229E (Fig. 2B). Together, these data indicate that a soluble compound distinct from, but present in the PG sample, is responsible for reducing CoV infectivity.

\section{Mass spectrometry identifies the inhibitor as surfactin.}

Having isolated the inhibitory molecule, we utilized mass spectrometry to determine its identity. Unwashed B. subtilis PG and ethanol supernatants were analyzed using MALDI-TOF/TOF mass spectrometry (Fig. 2C). In the PG samples, three prominent peaks were observed with masses of 1010.5, 1058.7, and 1238.6 (Fig. 2C). While all of these peaks were present in the ethanol supernatant, the compound with a mass 1058.7 was enriched nearly 10 -fold (Fig. 2D). Further analysis of this peak by fragmentation produced a spectrum matching that of the cyclic lipopeptide surfactin (10) a potent biosurfactant produced naturally by $B$. subtilis and shown previously to have antimicrobial and antiviral properties (11, 12) (Fig 2E, for structure see Fig 6A). Given its abundance, enrichment in the ethanol wash, as well as its described antiviral properties, we concluded that surfactin likely conferred the $B$. subtilis PG with the ability to 21 interfere with CoV infection.

\section{Reduction of CoV infectivity by surfactin is temperature- and dose-dependent}

23 To confirm its inhibitory effect, we characterized the ability of purified surfactin to reduce CoV 24 infectivity. HCoV-229E, MERS-CoV, or SARS-CoV were treated with PBS or surfactin at either $25 \mathrm{RT}, 32^{\circ} \mathrm{C}$, or $37^{\circ} \mathrm{C}$. For all three $\mathrm{CoVs}$, surfactin reduced infectivity after treatment at $37^{\circ} \mathrm{C}$, with 26 a near complete loss of infectious virus (Fig. $3 \boldsymbol{A}-\boldsymbol{C}$ ). Similar to $B$. subtilis $P G$, the degree of 
1 reduction varied based on incubation temperature and varied between the CoVs (Fig. $3 \boldsymbol{A}-\boldsymbol{C}$ ). To

2 further characterized the kinetics of inhibition, HCoV-229E and MERS-CoV were treated with

3 surfactin at $4^{\circ} \mathrm{C}, \mathrm{RT}, 32^{\circ} \mathrm{C}$, or $37^{\circ} \mathrm{C}$ and sampled over a time-course (Fig. $3 \boldsymbol{D}-\boldsymbol{E}$ ). At both $32^{\circ} \mathrm{C}$

4 and $37^{\circ} \mathrm{C}$, surfactin rapidly reduced $\mathrm{HCoV}-229 \mathrm{E}$ and MERS-CoV, with a near complete loss of

5 infectivity after two hours of treatment (Fig. 3D-E). In contrast RT incubation reduced CoV

6 infectivity more slowly and, surfactin's effects were ablated at $4^{\circ} \mathrm{C}$. We also observed dose

7 dependent changes in surfactin activity against HCoV-229E, MERS-CoV, and SARS-CoV (Fig.

$83 F$. Interestingly, higher concentrations of surfactin were required for inhibition of HCoV-229E

9 when compared to either SARS-CoV or MERS-CoV, whose inhibition curves were nearly

10 identical. Together, these data indicate that both temperature and dose impact surfactin's

11 inhibitory effects.

\section{Surfactin reduces CoV infectivity by disrupting the structural integrity of viral particles.}

13 Prior studies with surfactin offers two mechanisms for virucidal activity: disruption of the viral

14 membrane or inhibition of host-virus membrane fusion (11-13). To determine if virion integrity was maintained, we performed RNase I protection assays. Following surfactin treatment, particles were exposed to RNase I to digest exposed viral RNA; samples were subsequently extracted for RNA and relative viral RNA determined by quantitative reverse transcription real time PCR (RT-qPCR). Increasing surfactin concentrations correlated with a decrease in viral RNA and viral titer for both HCoV-229E (Fig. 4A) and MERS-CoV (Fig. 4B). These results indicate that disruption of virion integrity is the primary mechanism by which surfactin inhibits CoV infection. To confirm these results, we performed transmission electron microscopy (TEM) on HCoV-229E treated with surfactin or PBS. In PBS-treated samples, numerous intact HCoV-

23 229E particles could be visualized (Fig. 4C-D); in contrast, no viral particles were found in any 24 of the surfactin treated samples (Fig. 4D). Taken together these results demonstrate that 25 surfactin inhibits CoV infection primarily through the disruption of viral particles.

\section{In vivo characterization of surfactin on CoV infection}


1 With no approved therapeutics (14), emerging, zoonotic CoVs pose a significant threat to public

2 health $(15,16)$. Therefore, we wanted to examine the potential of surfactin to treat infections in

3 vivo. We tested whether direct treatment of the inoculum reduced in vivo infection and disease.

4 SARS-CoV $\left(10^{4}\right.$ plaque forming units (PFU)) was treated with PBS or surfactin and used to

5 infect BALB/c mice intranasally (IN). Mice were monitored over 4 days for weight loss and

6 lethality, with lung titers determined at 2- and 4-days post infection. As expected, animals

7 infected with PBS treated virus experienced rapid weight loss and exhibited high lung titers at

8 both 2- and 4-days post infection (Fig. 5A). In contrast, mice infected with surfactin treated

9 SARS-CoV lost no weight and no infectious virus was detected in their lungs (Fig. 5B).

Additionally, mock infected mice receiving surfactin alone demonstrated no signs of disease or weight loss, suggesting that surfactin treatment alone does not have any pathological effects

\section{2 (Fig. 5A).}

13 To examine therapeutic potential, we next evaluated if pretreatment with surfactin could reduce respiratory CoV disease. BALB/c mice were treated IN with $50 \mu$ of either PBS control or surfactin daily, starting 18 hours prior to infection, and continuing over the first two days of infection. Mice were subsequently infected with $10^{4}$ PFU of SARS-CoV (MA15) and monitored for weight loss and lethality, with lung titer determined at 2 and 4 days post-infection. In contrast to the surfactin-treated inoculum, prophylactic surfactin treatment had no effect on weight loss (Fig. 5C) or viral titer in the lung (Fig. 5D). These results indicate that prophylactic surfactin treatment by this route does not reduce SARS-CoV disease in this mouse model.

\section{Effects of other cyclic lipopeptides on CoV infectivity.}

22 Surfactin belongs to a family of 80 natural antibiotic compounds referred to as cyclic 23 lipopeptides (CLPs) (11). While structurally diverse, all CLPs share two key features: a non24 polar hydrocarbon tail and a non-ribosomally produced peptide ring $(11,12)$. While many CLPs

25 have been found to be antifungal and antibacterial, antiviral properties have not been described 26 except for surfactin, $(11,12)$. Therefore, we tested six CLPs for the ability to reduce CoV 
1 infectivity (Fig. 6A). Despite similar biochemical structures, none of the CLPs tested had a

2 significant effect on HCoV-229 or MERS-CoV infection (Fig. 6B). These results suggest that

3 unique features allow surfactin to reduce CoV infectivity.

\section{Surfactin broadly reduces viral infectivity}

5 With its potent antiviral properties against CoVs, we wanted to test surfactin's effect against

6 other highly pathogenic viruses. Given its ability to disrupt virion integrity, we focused on

7 enveloped viruses from diverse families including two Influenza A strains (H1N1, H3N2), Zika

8 Virus (ZIKV), Dugbe Virus (DUGV), Nipah Virus (NiV), Crimean-Congo Hemorrhagic Fever

9 Virus (CCHFV), Chikungunya Virus (CHIKV), Mayaro virus, Una virus, and Ebola virus (EBOV).

10 As a negative control, we tested the non-enveloped Coxsackievirus B3 (CVB3). Each virus was

11 treated with either PBS or surfactin and viral infectivity determined. As expected, surfactin had

12 no effect on the non-enveloped CVB3 (Fig. 6C). In contrast, surfactin significantly reduced

13 infectivity in each of the enveloped viruses (Fig. 6C), but the magnitude of effect was not

14 uniform. Most enveloped viruses were reduced either below their limit of detection or greater

15 than 100,000-fold. In contrast, Mayaro, both influenza strains, and EBOV exhibited some

16 resistance, having their infectious titer reduced only $2.6,2.7,2.4$, and 1.6 logs, respectively.

17 These data suggest that while surfactin treatment broadly reduced the infectivity of enveloped

18 viruses, factors beyond the mere presence or absence of an envelope may govern overall

19 sensitivity. 


\section{Discussion}

2 In this study, we explored the relationship between bacterial components and CoV infection.

3 While initially predicting enhanced infection, treatment with $B$. subtilis PG reduced CoV

4 infectivity, while envelope components from other bacteria had no effect. Separating the

5 inhibitory effect using solvent washes, we used mass spectrometry to identify that the CLP

6 surfactin was responsible for reduced CoV infectivity and disruption of virion integrity.

7 Unfortunately, despite efficacy against the inoculum, prophylactic surfactin treatment prior to

8 infection had no effect on CoV related disease in vivo. Notably, other CLPs had no effect on

9 CoV infectivity despite having similar biochemical structures. Finally, we found that surfactin

10 treatment was efficacious against many enveloped viruses in vitro including IAV strains H1N1

11 and H3N2, ZIKV, DUGV, NiV, CCHFV, CHIKV, Una, Mayaro, and EBOV. Together, these data

12 demonstrate that surfactin is a potent virucide and highlight that interactions with bacterial

13 derived compounds can also negatively modulate virus infection.

14 Over the last two decades, surfactin has been shown to be anti-bacterial, anti-fungal, and anti-

15 viral $(11,12,17-19)$. Mechanistically, surfactin's broad anti-microbial efficacy has been linked to

16 disruption of lipid membranes (13). However, more recently, researchers described surfactin's

17 efficacy against the animal CoV porcine epidemic diarrhea virus (PEDV), and suggested that

18 surfactin inhibited viral-host membrane fusion (19). In contrast to the PEDV results, we found

19 that surfactin treatment disrupted virion integrity, exposing the viral RNA to RNase I mediated

20 degradation (Fig. 4A-B). Transmission electron microscopy (TEM) confirmed the absence of

21 intact virions in surfactin treated samples (Fig. 4C). Thus, while both PEDV and human CoVs

22 are sensitive to surfactin treatment, infectivity reduction may be the result of different

23 mechanisms due to differences the surfactin dose, virion composition, tissue environment

24 (respiratory vs enteric), or other factor. 
1 Similar to the question of mechanism, in vivo efficacy of surfactin also varied between PEDV

2 and human CoVs. While surfactin ablates SARS-CoV disease when treating the inoculum,

3 prophylactic treatment was not protective. Why surfactin failed to protect mice against SARS-

$4 \mathrm{CoV}$ is puzzling, given that efficacy of prophylactic oral surfactin treatment against PEDV

5 disease (19). One explanation is the physical environment of the respiratory and gastrointestinal

6 tracts differs significantly, making intranasal (IN) surfactin administration ineffective due to tissue

7 specific differences. Thus, while oral surfactin administration may be effective at delivering

8 surfactin to infected gastrointestinal tissue, IN administration may not be as effective,

9 particularly in the lower parts of the lung. Alternative delivery methods such as the inhalation of

10 an aerosolized surfactin may overcome these problems. Additionally, several surfactin

11 derivatives exist and may enhance its virucidal activity in the context of respiratory infection in

12 vivo $(11,12)$.

13 In addition to CoVs, we examined surfactin's virucidal efficacy against other enveloped viruses,

14 discovering broad efficacy, but wide variation. While all tested enveloped viruses were sensitive

15 to surfactin treatment, IAV strains H1N1 and H3N2, Mayaro, and EBOV demonstrated a degree

16 of resistance. These data suggest that factors beyond the mere presence of a viral envelope

17 regulate surfactin efficacy. One possible factor is the lipid content of the viral envelope. Previous

18 studies have shown that membranes enriched in cholesterol and phosphatidylethanolamines

19 (PE) are resistant to surfactin permeabilization, while membranes containing

20 phosphatidylcholines (PC) are more sensitive (20). The envelope of Influenza A viruses have

21 been reported to be enriched for both cholesterol and PE (21), providing support for this

22 hypothesis. Unfortunately, the lipid content of the other viruses tested have not been

23 determined, preventing direct comparison. Nevertheless, some broad observations are worth

24 mentioning. CoVs, ZIKV, and bunyaviruses (CCHFV and DUGV) derive their envelopes from

25 either the Golgi Apparatus and Endoplasmic Reticulum, organelles enriched in surfactin 
1 sensitive PC (22). NiV (23), IAV (24), EBOV (25) are thought to derive their envelopes from

2 lipid rafts of the plasma membrane, which could specify their lipid content and thus surfactin

3 sensitivity. Alphaviruses such as CHIKV, Mayaro, and Una also bud form the plasma

4 membrane, though neither the lipid content, nor the involvement of lipid rafts has been explored

5 (26). Together, these observations suggest the lipid content of enveloped viruses may explain

6 their differential sensitivity to surfactin.

7 The failure of other CLPs to reduce CoV infectivity is also surprising, given the structural

8 similarity to surfactin. In particular, Iturin A is both biochemically similar to surfactin and has

9 also been reported to disrupt lipid membranes (Fig. 6A) (11). A possible explanation involves

differences in their mechanisms of action. Surfactin penetrates lipid layers, alone solubilizing

11 and permeabilizing them (13). In contrast, Iturin A must interact with sterol components to cause

12 membrane permeabilization, explaining its broad anti-fungal, but only selective antibacterial

13 activity (11). However, Iturin $A$ is also quite hemolytic $(11,12)$, making it unclear why the

14 membranes of enveloped viruses grown in mammalian cells would not also be susceptible to

15 this mechanism, due to the presence of sterols. Compounding this mechanistic uncertainty,

16 daptomycin's permeabilization of membranes requires no such interaction, but CoVs are

17 resistant to its effects as well (27) (Fig. 6B). The results argue that surfactin possesses unique

18 properties conferring its virucidal activity. Surfactin is known to adopt a unique $\beta$-sheet like

19 "horse-saddle" conformation, which may facilitate membrane permeabilization (13). Molecular

20 dynamics simulations suggest temperature directly regulates the openness of the horse saddle

21 structure and may explain why surfactin's virucidal activity is also temperature sensitive (28). In

22 total, these results highlight our poor understanding of membrane disruption by CLPs and

23 argues that biochemical studies of these compounds inhibition of enveloped viruses are needed. 
1 While the microbiome has historically been thought to serve a protective role against pathogens

$2(1,2)$, recent studies with viruses complicate this view. Studies with poliovirus demonstrated

3 that the presence of commensal bacteria is necessary for oral poliovirus infection in mice (29).

4 Similar findings with other enteric viruses suggest that utilizing bacterial components is a

5 common approach. In contrast, our results add further complexity, demonstrating that surfactin,

6 a secondary metabolite of $B$. subtilis, can potently reduce CoV infectivity. Though $B$. subtilis is

7 not generally part of the human microbiome (30), it is often used as an intestinal probiotic and

8 has been found to transiently persist in the gut (31). Additionally, surfactin-like molecules are

9 produced by a broad array of bacterial species (11, 32-34). For example, the novel surfactin like

10 CLP Coryxin was recently found to be produced by Corynebacterium xerosis, a common

11 member of the respiratory microbiome (34). These facts suggest that microbial components

12 typically thought to work against bacterial competitors could also potentially disrupt viral

13 infection. Thus, as the relationship between the microbiome and viral infections is further

14 explored, the role bacterial metabolites such as surfactin and other CLPs play in modulating

15 infection must be considered in viral disease. Overall, these results highlight the dynamic

16 microbial environment and its potential to impact viral pathogenesis as well as identify novel

17 inhibitory factors for therapeutic use. 


\section{Materials and Methods}

2 Viruses, cells, and in vitro infection. HCoV-229E, provided by the World Reference Center

3 for Emerging Viruses and Arboviruses (WRCEVA), was propagated on HUH7 cells grown in

$4 \operatorname{DMEM}$ (Gibco), 10\% fetal bovine serum (Hyclone), and 1\% antibiotic-antimycotic (A/A) (Gibco).

5 Titration was performed by $\mathrm{TCID}_{50}$ in $\mathrm{HUH7}$ cells and calculated by the Spearman-Karber

6 Method. MERS-CoV (EMC-2012 strain) (35) and recombinant SARS-CoV (MA15) (36) were

7 titrated and propagated on VeroCCL81 and VeroE6 cells, respectively, grown in DMEM with 5\%

8 fetal bovine serum and 1\% A/A. Standard plaque assays were used for SARS- and MERS-CoV

9 (37, 38). Cocksackievirus B3 (39), chikungunya (40), Nipah (41), Dugbe (42), Zika (43),

10 Crimean-Congo hemorrhagic fever (42), Influenza A H1N1(A/California/04/09) H3N2

11 (A/Panama/2007/99) (44), and Ebola viruses (45) were propagated and quantitated via standard

12 methods. All experiments involving infectious virus were conducted at the University of Texas

13 Medical Branch (Galveston, TX) in approved biosafety level 2, 3, or 4 (BSL) laboratories and

14 animal facilities, with routine medical monitoring of staff.

Treatment with bacterial surface components and cyclic lipopeptides. CoVs were diluted

$10 \% \mathrm{vol} / \mathrm{vol}$ in solutions with final concentrations of $1 \mathrm{mg} / \mathrm{ml}$ (PG and LPS) or $100 \mu \mathrm{g} / \mathrm{ul}$ (CLPs)

17 unless otherwise specified in the text. For alcohol wash experiments, samples instead diluted

$185 \% \mathrm{vol} / \mathrm{vol}$. Treated samples were then incubated for 2 hours at $37^{\circ} \mathrm{C}$, after which they were

19 titrated. Bacterial components were purchased from Sigma-Aldrich; lipopolysaccharides from

20 Escherichia coli (L4130), peptidoglycan Bacillus subtilis (69554), Staphylococcus aureus

21 (77140), Streptomyces spp. (79682), and Micrococcus luteus (53243). Peptidoglycan from

22 Escherichia coli (PGN-EB) was purchased from Invivogen. For each surface component, stock

23 solutions were created by suspending the component in PBS and stored and $-20^{\circ} \mathrm{C}$. The cyclic

24 lipopeptides surfactin (S3523), iturin A (11774), fengycin (SMB00292), polymyxin B (P1004), 
1 colisitn (C4461), ramoplanin (R1781), and daptomycin (D2446) were also purchased from

2 Sigma-Aldrich.

3 Mass spectrometry. Stock peptidoglycan was centrifuged at 15,000g for 1 minute in a table top

4 centrifuge and the insoluble PG fraction was then resuspended in $100 \%$ ethanol. Following a 5-

5 minute incubation at room temperature, samples were centrifuged, and the supernatant and

6 insoluble fractions were used for treatment of viruses or delivered to the mass spectrometry

7 core facility. $1 \mu \mathrm{l}$ of peptidoglycan was combined $1: 1$ with a $10 \mathrm{mg} / \mathrm{ml}$ a-cyano-4-

8 hydroxycinnamic acid (60\% acetonitrile) and spotted onto MALDI targets. All MALDI-MS

9 experiments were performed using a 5800 MALDI-TOF/TOF (Applied Biosystems). The MS 10 data were acquired using the reflectron detector in positive mode (700-4500 Da, $1900 \mathrm{Da}$ focus 11 mass) using 300 laser shots (50 shots per sub-spectrum). Collision induced dissociation tandem

12 MS spectra were acquired using $1 \mathrm{kV}$ of collision energy. Fragmentation data was analyzed

13 manually to determine structural information.

14 Transmission Electron Microscopy. HCoV-229E virions were visualized by transmission 15 electron microscopy (TEM) through negative staining with 2\% Uranyl acetate (46). Briefly, 200 16 mesh formvar carbon-coated copper grids (FCF200-CU) from Electron Microscopy Sciences 17 were treated for 20 minutes with HCoV-229E samples. Excess sample solution was then wicked 18 off with filter paper, and each grid was then stained for 45 seconds with $2 \%$ Uranyl-acetate 19 solution. Excess stain was again wicked off with filter paper. Grids were then dried and 20 visualized on a Philips CM100 TEM Electron microscope. Images were recorded with a Gatan 21 Orius SC200 CCD camera. In order to ensure even counting, 10 pictures were taken on 3 22 different cells on each grid. No more than 10 minutes were allotted for looking for virus in each 23 cell.

24 RNase I protection assay. Assays were performed in accordance with standard protocols 25 described previously (47). Briefly, Samples were treated either with or without 250U RNase I for 
130 minutes. To halt RNA digestion and inactivate RNase I, 2 times volume of Viral RNA Buffer

2 from Zymo Research (R1034-1-100) with 2-mercaptoethanol was added. RNA was then

3 extracted using the Quick-RNA Viral Kit from Zymo Research (R1035). RNA was then

4 converted into cDNA using the iScript cDNA synthesis Kit (170-8891) from Bio-Rad.

5 Quantitative real time PCR was performed using SsoAdvanced Universal SYBR Green

6 Supermix (172-5271) from Bio-Rad. HCoV-229E specific primer sequences were Forward: 5-

7 TGACATTCGCGACTACAAGC-3 and Reverse: 5-TAACGGTGGTTTGGCTTTTC-3. MERS-CoV

8 specific primer sequences were Forward: 5-TCGCTTGGCAAATGAGTGTG-3 and Reverse: 5-

9 ACATTAGCAGTTGTCGCCTG-3.

Statistical analysis. All statistical comparisons in this manuscript involved the comparison

11 between 2 groups, untreated control virus and peptidoglycan/surfactin treated virus. Thus,

12 significant differences in viral titer, TEM counts, RNA levels, and weight loss were determined

13 by the unpaired two-tailed students T-Test.

14 Ethic Statement. This study was carried out in accordance with the recommendations for care

15 and use of animals by the Office of Laboratory Animal Welfare, National Institutes of Health.

16 The Institutional Animal Care and Use Committee (IACUC) of University of Texas Medical

17 Branch (UTMB) approved the animal studies under protocol 1711065 and 1707046.

18 Mice and in vivo infection. Ten-week old BALB/c mice were purchased from Charles River

19 Laboratories and maintained in Sealsafe ${ }^{T M}$ HEPA-filtered air in/out units. Animals were

20 anesthetized with isoflurane and infected intranasally (IN) with $10^{4}$ PFU in $50 \mu$ of phosphate-

21 buffered saline (PBS). Infected animals were monitored for weight loss, morbidity, and clinical

22 signs of disease, and lung titers were determined as described previously (48). For experiments

23 involving prophylactic treatment with surfactin, $50 \mu \mathrm{l}$ of $100 \mu \mathrm{g} / \mathrm{ml}$ surfactin-PBS was IN

24 administered to anesthetized animals 18 hours prior to infection with additional treatments on 
day 0 , day 1 , and day 2 . Infected animals were weighed daily, and lungs collected 2 and 4 days post-infection for downstream analysis by plaque assay.

Acknowledgements. Research was supported by grants from NIA and NIAID of the NIH the University of Texas System to VDM and trainee funding provided by the McLaughlin

7 Endowment at UTMB.

\section{References}

1. Man WH, de Steenhuijsen Piters WA, Bogaert D. 2017. The microbiota of the respiratory tract: gatekeeper to respiratory health. Nat Rev Microbiol 15:259-270.

2. Thaiss CA, Zmora N, Levy M, Elinav E. 2016. The microbiome and innate immunity. Nature 535:65-74.

3. Karst SM. 2016. The influence of commensal bacteria on infection with enteric viruses. Nat Rev Microbiol 14:197-204.

4. Berger AK, Yi H, Kearns DB, Mainou BA. 2017. Bacteria and bacterial envelope components enhance mammalian reovirus thermostability. PLoS Pathog 13:e1006768.

5. Drexler JF, Corman VM, Drosten C. 2014. Ecology, evolution and classification of bat coronaviruses in the aftermath of SARS. Antiviral Res 101:45-56.

6. Gralinski LE, Menachery VD, Morgan AP, Totura AL, Beall A, Kocher J, Plante J, Harrison-Shostak DC, Schäfer A, Pardo-Manuel de Villena F, Ferris MT, Baric RS. 2017. Allelic Variation in the TollLike Receptor Adaptor Protein. G3 (Bethesda) 7:1653-1663.

7. Totura AL, Whitmore A, Agnihothram S, Schäfer A, Katze MG, Heise MT, Baric RS. 2015. Toll-Like Receptor 3 Signaling via TRIF Contributes to a Protective Innate Immune Response to Severe Acute Respiratory Syndrome Coronavirus Infection. MBio 6:e00638-15.

8. Sheahan T, Morrison TE, Funkhouser W, Uematsu S, Akira S, Baric RS, Heise MT. 2008. MyD88 is required for protection from lethal infection with a mouse-adapted SARS-CoV. PLoS Pathog 4:e1000240.

9. Vollmer W, Blanot D, de Pedro MA. 2008. Peptidoglycan structure and architecture. FEMS Microbiol Rev 32:149-67.

10. Yang H, Li X, Yu H, Shen Z. 2015. Identification of lipopeptide isoforms by MALDI-TOF-MS/MS based on the simultaneous purification of iturin, fengycin, and surfactin by RP-HPLC. Anal Bioanal Chem 407:2529-42.

11. Cochrane SA, Vederas JC. 2016. Lipopeptides from Bacillus and Paenibacillus spp.: A Gold Mine of Antibiotic Candidates. Med Res Rev 36:4-31.

12. Meena KR, Kanwar SS. 2015. Lipopeptides as the antifungal and antibacterial agents: applications in food safety and therapeutics. Biomed Res Int 2015:473050.

13. Seydlová G, Svobodová J. 2008. Review of surfactin chemical properties and the potential biomedical applications. Central European Journal of Medicine 3:123-133. 
14. Mustafa S, Balkhy H, Gabere MN. 2018. Current treatment options and the role of peptides as potential therapeutic components for Middle East Respiratory Syndrome (MERS): A review. J Infect Public Health 11:9-17.

15. World Health Organization. 2004. WHO SARS Risk Assessment and Preparedness Framework. WHO Press, Geneva.

16. World Health Organization. 2017. WHO MERS-CoV Global Summary and Assessment of Risk. WHO Press, Geneva.

17. Wang $\mathrm{X}, \mathrm{Hu} \mathrm{W}$, Zhu L, Yang Q. 2017. Bacillus subtilis and surfactin inhibit the transmissible gastroenteritis virus from entering the intestinal epithelial cells. Biosci Rep 37.

18. Pang $X$, Zhao J, Fang $X$, Liu H, Zhang $Y$, Cen S, Yu L. 2017. Surfactin derivatives from Micromonospora sp. CPCC 202787 and their anti-HIV activities. J Antibiot (Tokyo) 70:105-108.

19. Yuan L, Zhang S, Wang Y, Li Y, Wang X, Yang Q. 2018. Surfactin Inhibits Membrane Fusion during Invasion of Epithelial Cells by Enveloped Viruses. J Virol 92.

20. Carrillo C, Teruel JA, Aranda FJ, Ortiz A. 2003. Molecular mechanism of membrane permeabilization by the peptide antibiotic surfactin. Biochim Biophys Acta 1611:91-7.

21. Ivanova PT, Myers DS, Milne SB, McClaren JL, Thomas PG, Brown HA. 2015. Lipid composition of viral envelope of three strains of influenza virus - not all viruses are created equal. ACS Infect Dis 1:399-452.

22. van Meer G, Voelker DR, Feigenson GW. 2008. Membrane lipids: where they are and how they behave. Nat Rev Mol Cell Biol 9:112-24.

23. Harrison MS, Sakaguchi T, Schmitt AP. 2010. Paramyxovirus assembly and budding: building particles that transmit infections. Int J Biochem Cell Biol 42:1416-29.

24. Rossman JS, Lamb RA. 2011. Influenza virus assembly and budding. Virology 411:229-36.

25. Bavari S, Bosio CM, Wiegand E, Ruthel G, Will AB, Geisbert TW, Hevey M, Schmaljohn C, Schmaljohn A, Aman MJ. 2002. Lipid raft microdomains: a gateway for compartmentalized trafficking of Ebola and Marburg viruses. J Exp Med 195:593-602.

26. Brown RS, Wan JJ, Kielian M. 2018. The Alphavirus Exit Pathway: What We Know and What We Wish We Knew. Viruses 10.

27. Taylor SD, Palmer M. 2016. The action mechanism of daptomycin. Bioorg Med Chem 24:62536268.

28. She AQ, Gang HZ, Mu BZ. 2012. Temperature influence on the structure and interfacial properties of surfactin micelle: a molecular dynamics simulation study. J Phys Chem B 116:12735-43.

29. Kuss SK, Best GT, Etheredge CA, Pruijssers AJ, Frierson JM, Hooper LV, Dermody TS, Pfeiffer JK. 2011. Intestinal microbiota promote enteric virus replication and systemic pathogenesis. Science 334:249-52.

30. de Steenhuijsen Piters WA, Sanders EA, Bogaert D. 2015. The role of the local microbial ecosystem in respiratory health and disease. Philos Trans R Soc Lond B Biol Sci 370.

31. Jeżewska-Frąckowiak J, Seroczyńska K, Banaszczyk J, Jedrzejczak G, Żylicz-Stachula A, Skowron PM. 2018. The promises and risks of probiotic Bacillus species. Acta Biochim Pol 65:509-519.

32. Burja AM, Abou-Mansour E, Banaigs B, Payri C, Burgess JG, Wright PC. 2002. Culture of the marine cyanobacterium, Lyngbya majuscula (Oscillatoriaceae), for bioprocess intensified production of cyclic and linear lipopeptides. J Microbiol Methods 48:207-19.

33. Morikawa M, Daido H, Takao T, Murata S, Shimonishi Y, Imanaka T. 1993. A new lipopeptide biosurfactant produced by Arthrobacter sp. strain MIS38. J Bacteriol 175:6459-66.

34. Dalili D, Amini M, Faramarzi MA, Fazeli MR, Khoshayand MR, Samadi N. 2015. Isolation and structural characterization of Coryxin, a novel cyclic lipopeptide from Corynebacterium xerosis NS5 having emulsifying and anti-biofilm activity. Colloids Surf B Biointerfaces 135:425-32. 
35. Kindler E, Jónsdóttir HR, Muth D, Hamming OJ, Hartmann R, Rodriguez R, Geffers R, Fouchier RA, Drosten C, Müller MA, Dijkman R, Thiel V. 2013. Efficient replication of the novel human betacoronavirus EMC on primary human epithelium highlights its zoonotic potential. MBio 4:e00611-12.

36. Roberts A, Deming D, Paddock CD, Cheng A, Yount B, Vogel L, Herman BD, Sheahan T, Heise M, Genrich GL, Zaki SR, Baric R, Subbarao K. 2007. A mouse-adapted SARS-coronavirus causes disease and mortality in BALB/C mice. PLoS Pathog 3:e5.

37. Sims AC, Tilton SC, Menachery VD, Gralinski LE, Schäfer A, Matzke MM, Webb-Robertson BJ, Chang J, Luna ML, Long CE, Shukla AK, Bankhead AR, Burkett SE, Zornetzer G, Tseng CT, Metz TO, Pickles R, McWeeney S, Smith RD, Katze MG, Waters KM, Baric RS. 2013. Release of severe acute respiratory syndrome coronavirus nuclear import block enhances host transcription in human lung cells. J Virol 87:3885-902.

38. Josset L, Menachery VD, Gralinski LE, Agnihothram S, Sova P, Carter VS, Yount BL, Graham RL, Baric RS, Katze MG. 2013. Cell host response to infection with novel human coronavirus EMC predicts potential antivirals and important differences with SARS coronavirus. MBio 4:e0016513.

39. Bradrick SS, Lieben EA, Carden BM, Romero JR. 2001. A predicted secondary structural domain within the internal ribosome entry site of echovirus 12 mediates a cell-type-specific block to viral replication. J Virol 75:6472-81.

40. Plante KS, Rossi SL, Bergren NA, Seymour RL, Weaver SC. 2015. Extended Preclinical Safety, Efficacy and Stability Testing of a Live-attenuated Chikungunya Vaccine Candidate. PLoS Negl Trop Dis 9:e0004007.

41. Freiberg AN, Worthy MN, Lee B, Holbrook MR. 2010. Combined chloroquine and ribavirin treatment does not prevent death in a hamster model of Nipah and Hendra virus infection. J Gen Virol 91:765-72.

42. Bente DA, Alimonti JB, Shieh WJ, Camus G, Ströher U, Zaki S, Jones SM. 2010. Pathogenesis and immune response of Crimean-Congo hemorrhagic fever virus in a STAT-1 knockout mouse model. J Virol 84:11089-100.

43. Widman DG, Young E, Yount BL, Plante KS, Gallichotte EN, Carbaugh DL, Peck KM, Plante J, Swanstrom J, Heise MT, Lazear HM, Baric RS. 2017. A Reverse Genetics Platform That Spans the Zika Virus Family Tree. MBio 8.

44. Rajsbaum R, Albrecht RA, Wang MK, Maharaj NP, Versteeg GA, Nistal-Villán E, García-Sastre A, Gack MU. 2012. Species-specific inhibition of RIG-I ubiquitination and IFN induction by the influenza A virus NS1 protein. PLoS Pathog 8:e1003059.

45. Bharaj P, Atkins C, Luthra P, Giraldo MI, Dawes BE, Miorin L, Johnson JR, Krogan NJ, Basler CF, Freiberg AN, Rajsbaum R. 2017. The Host E3-Ubiquitin Ligase TRIM6 Ubiquitinates the Ebola Virus VP35 Protein and Promotes Virus Replication. J Virol 91.

46. Berryman MA, Rodewald RD. 1990. An enhanced method for post-embedding immunocytochemical staining which preserves cell membranes. J Histochem Cytochem 38:15970.

47. Gilling DH, Kitajima M, Torrey JR, Bright KR. 2014. Mechanisms of antiviral action of plant antimicrobials against murine norovirus. Appl Environ Microbiol 80:4898-910.

48. Sheahan T, Whitmore A, Long K, Ferris M, Rockx B, Funkhouser W, Donaldson E, Gralinski L, Collier M, Heise M, Davis N, Johnston R, Baric RS. 2011. Successful vaccination strategies that protect aged mice from lethal challenge from influenza virus and heterologous severe acute respiratory syndrome coronavirus. J Virol 85:217-30. 
Figure 1: Peptidoglycan from Bacillus subtilis reduces coronavirus infectivity. (A) Bacterial envelope components such as LPS are bound to CoVs, increasing their thermostability (right) relative to untreated samples (left). (B) Relative infectivity of HCoV-229E $(n=4)$ and MERS-CoV ( $n=5)$ after treatment with PBS alone (black), LPS from Escherichia coli (grey), or PG from B. subtilis (green) following 2 hour incubation at $37^{\circ} \mathrm{C}$. (C) HCoV-299E (circles) and MERS-CoV (triangles) infectivity after treatment for 2 hours at $37^{\circ} \mathrm{C}$ with peptidoglycan from the indicated bacterial species ( $n=3$ ). (D) HCoV-229E and (E) MERS-CoV after treatment with PG

9 from $B$. subtilis at room temperature $(R T), 32^{\circ} \mathrm{C}$, and $37^{\circ} \mathrm{C}(n=3)$. For all dot plots, the centered bar represents the group mean while the error bars represent SD. P-values are based on the two-tailed Student's $t$ test as indicated: ${ }^{*} P<0.05,{ }^{* *} P<0.01,{ }^{* * *} P<0.001$.

Figure 2: Identification of surfactin from B. subtilis peptidoglycan. (A and B) PG from $B$. subtilis in a PBS solution was clarified, washed with the indicated solvents, and clarified again. Supernatants were decanted and retained, while the insoluble fractions were resuspended in PBS. The $(A)$ insoluble fraction and $(B)$ supernatants were then used to treat HCoV-229E and relative infectivity determined $(n=3)$. (C, D, and $E)$ Mass spectrometry performed was performed on PG (C) and ethanol wash (D). The peak corresponding to the molecular mass 1058 in the ethanol wash was then further fragmented $(E)$ to determine the identity of the molecule. Representative spectra shown. For all dot plots, the centered bar represents the group mean and error bars the SD.

Figure 3: Characterization of CoV inhibition by surfactin. (A) HCoV-229E, (B) MERS-CoV, and (C) SARS-CoV (MA15) were treated PBS alone (black) or surfactin (red) and at room 23 temperature $(\mathrm{RT}), 32^{\circ} \mathrm{C}$, and $37^{\circ} \mathrm{C}$ and infectivity determined $(\mathrm{n}=3)$. HCoV-229E (D) and MERS-

$24 \mathrm{CoV}(\mathrm{E})$ were treated for the indicated time at $4^{\circ} \mathrm{C}$ (light orange), $\mathrm{RT}$ (dark orange), $32^{\circ} \mathrm{C}$ (light 25 red), or $37^{\circ} \mathrm{C}$ (dark red) and infectivity determined ( $\mathrm{n}=3$ ). (F) HCoV-229E (blue), MERS-CoV 26 (orange), and SARS-CoV MA15 (green) were diluted over a range of concentrations of surfactin 
1 and viral infectivity determined $(n=3)$. For dot plots, each point represents the titer from an

2 independent experiment while the group mean is indicated by a line. Each point on the line

3 graph represents the group mean. All error bars represent SD. The two tailed students t-test

4 was used to determine $P$-values: ${ }^{*} P<0.05,{ }^{* *} P<0.01,{ }^{* *} P<0.001$.

5 Figure 4: Surfactin disrupts CoV structural integrity. (A) HCoV-229E and (B) MERS-CoV

6 were treated with the indicated concentrations of surfactin. Viral infectivity was then determined

7 (red) or samples were then treated with RNAse I, RNA extracted, and viral genome copy

8 number determined by RT-qPCR (black). (C and D) PBS or surfactin treated HCoV-229E

9 samples negatively stained and examined by TEM and virions counted $(n=3)$. Representative

10 micrograph shown in (C) while total counts are displayed in (D). Horizontal lines represent group

11 mean while error bars represent SD. Two-tailed students t-test determined significance: * $P<$ $0.05,{ }^{* *} P<0.01,{ }^{* * *} P<0.001$.

Figure 5: In vivo characterization of surfactin treatment on SARS-CoV infection. (A and B)

BALB/C mice were then intranasally infected with $10^{4}$ PFU of PBS (black) or surfactin (red)

treated SARS-CoV MA15 and (A) monitored for weight loss over 4 days. Dotted lines and triangles represent mock infected animals with PBS alone (black) or surfactin alone (red). (B)

17 Lung tissue was harvested and viral titer determined at day 2 and day $4 . \mathrm{n}=4$ for all infected groups, $n=2$ for mock groups. ( $C$ and $D)$ BALB/C mice were pretreated intranasally with $50 \mu l$ of either PBS (black) or surfactin in PBS (red). 18 hours later, BALB/C mice were infected with $10^{4}$ PFU of SARS-CoV (MA15) and (C) monitored for weight loss over 4 days. (D) Lung titer

21 determined 2- $(n=5)$ and 4-days post infection $(n=10)$. Dots on line graphs and bars on bar 22 graphs represent the group mean. ND indicates that no titers were detected. All error bars 23 represent SD. P-values were calculated using the two-tailed student's t-test, with: * $P<0.05$, ** $24 P<0.01,{ }^{* * *} P<0.001$. 
1 Figure 6: Surfactin, but not other cyclic lipopeptides, broadly reduce the infectivity of

2 enveloped viruses. (A) Biochemical models of each of the seven cyclic lipopeptides tested.

3 The number of amino acids present in the cyclic ring are shown in parentheses. (B) HCoV-229E

4 (blue) and MERS-CoV (grey) were treated with PBS or the indicated cyclic lipopeptides in PBS

5 and incubated for 2 hours at $37^{\circ} \mathrm{C}$. Viral infectivity was then determined $(n=3)$. (C) The indicated

6 viruses were diluted PBS (black) or surfactin (red), incubated for 2 hours at $37^{\circ} \mathrm{C}$, and viral

7 infectivity determined $(n=3)$. Viruses are abbreviated as follows: Coxsackievirus (CVB3), Dugbe

8 (DUGV), Crimean-Congo hemorrhagic fever (CCHFV), Zika (ZIKV), Nipah (NiV), and

9 Chikungunya (CHIKV), Una, Mayaro, Influenza A strains H1N1 and H3N2, and Ebola (EBOV).

10 Bar graph bars represent the group means. Error bars represent SD. ND indicates that no titers

11 were detected. The student's t-test was used to calculate $p$-values, with: * $P<0.05$, ${ }^{* *} P<0.01$, ${ }^{* * *} P<0.001$. 

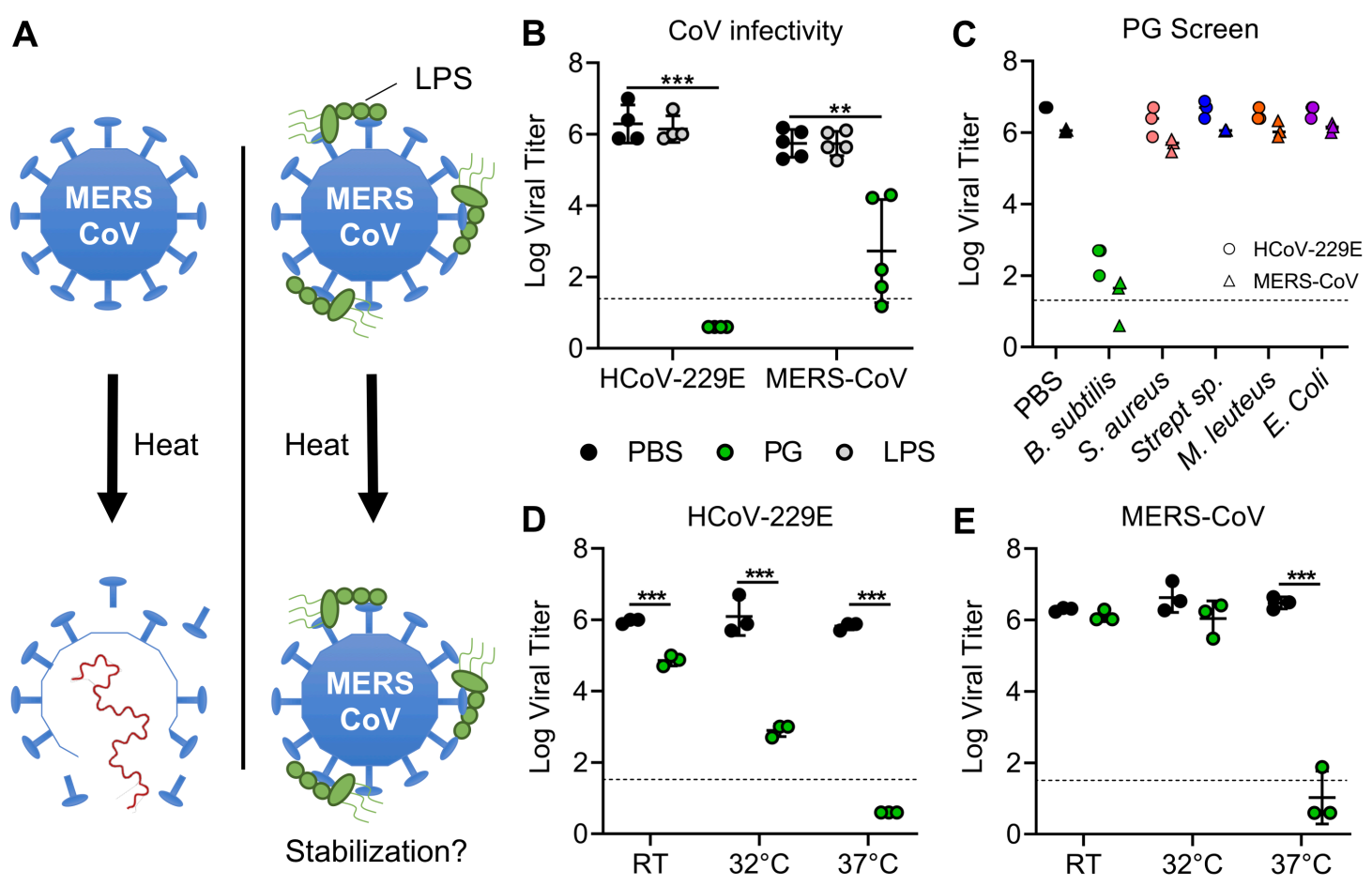

Figure 1: Peptidoglycan from Bacillus subtilis reduces coronavirus infectivity. (A) Bacterial envelope components such as LPS are bound to CoVs, increasing their thermostability (right) relative to untreated samples (left). (B) Relative infectivity of HCoV-229E $(n=4)$ and MERS-CoV $(n=5)$ after treatment with PBS alone (black), LPS from Escherichia coli (grey), or PG from $B$. subtilis (green) following 2 hour incubation at $37^{\circ} \mathrm{C}$. (C) HCoV-299E (circles) and MERS-CoV (triangles) infectivity after treatment for 2 hours at $37^{\circ} \mathrm{C}$ with peptidoglycan from the indicated bacterial species $(n=3)$. (D) HCoV229E and (E) MERS-CoV after treatment with PG from $B$. subtilis at room temperature $(\mathrm{RT}), 32^{\circ} \mathrm{C}$, and $37^{\circ} \mathrm{C}(\mathrm{n}=3)$. For all dot plots, the centered bar represents the group mean while the error bars represent SD. P-values are based on the two-tailed Student's $t$ test as indicated: ${ }^{*} P<0.05,{ }^{* *} P<0.01,{ }^{* * *} P<0.001$. 

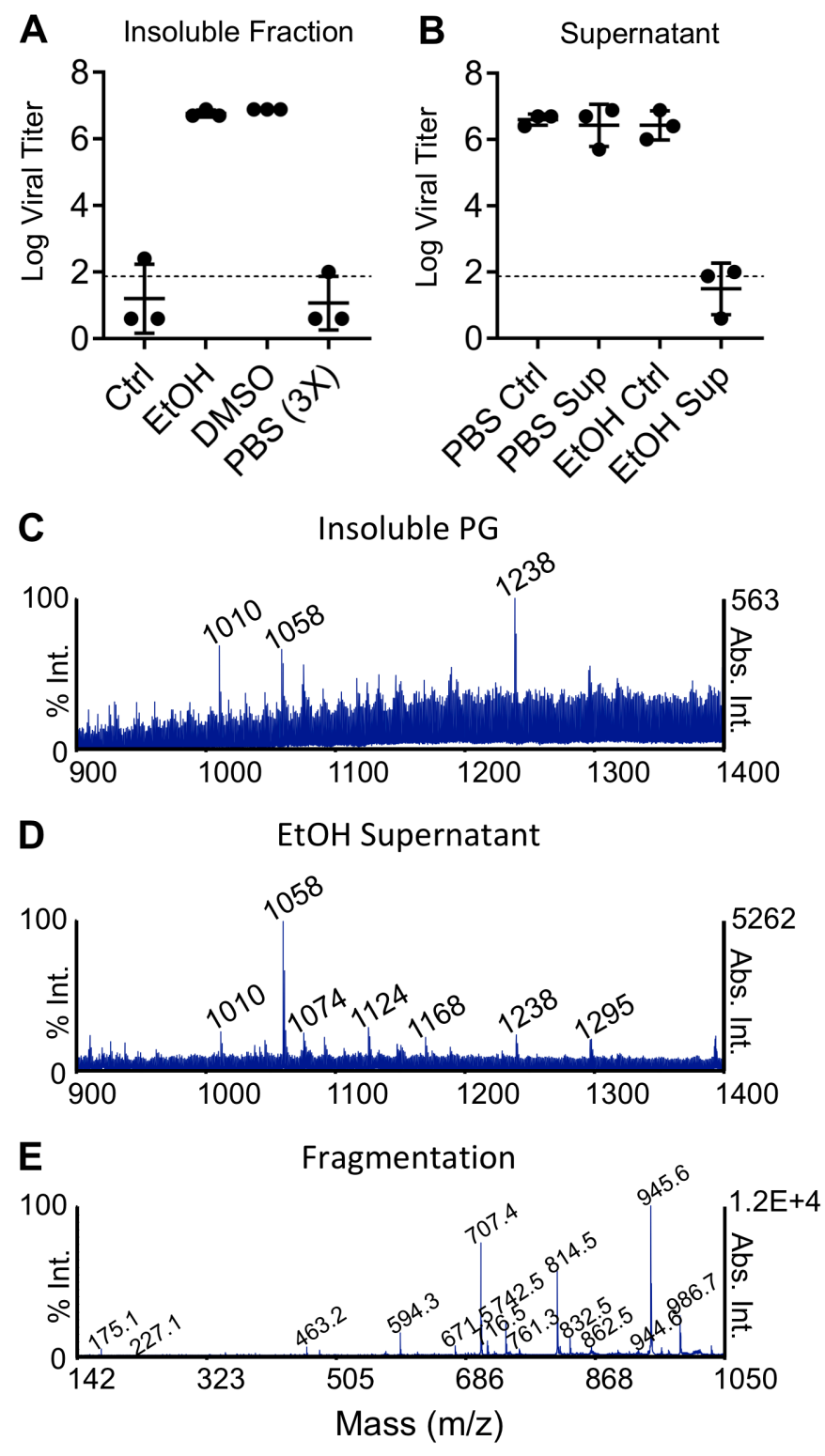

Figure 2: Identification of surfactin from $B$. subtilis peptidoglycan. ( $A$ and $B$ ) PG from $B$. subtilis in a PBS solution was clarified, washed with the indicated solvents, and clarified again. Supernatants were decanted and retained, while the insoluble fractions were resuspended in PBS. The $(A)$ insoluble fraction and $(B)$ supernatants were then used to treat HCoV-229E and relative infectivity determined $(n=3)$. (C, D, and E) Mass spectrometry performed was performed on PG (C) and ethanol wash (D). The peak corresponding to the molecular mass 1058 in the ethanol wash was then further fragmented $(\mathrm{E})$ to determine the identity of the molecule. Representative spectra shown. For all dot plots, the centered bar represents the group mean and error bars the SD. 

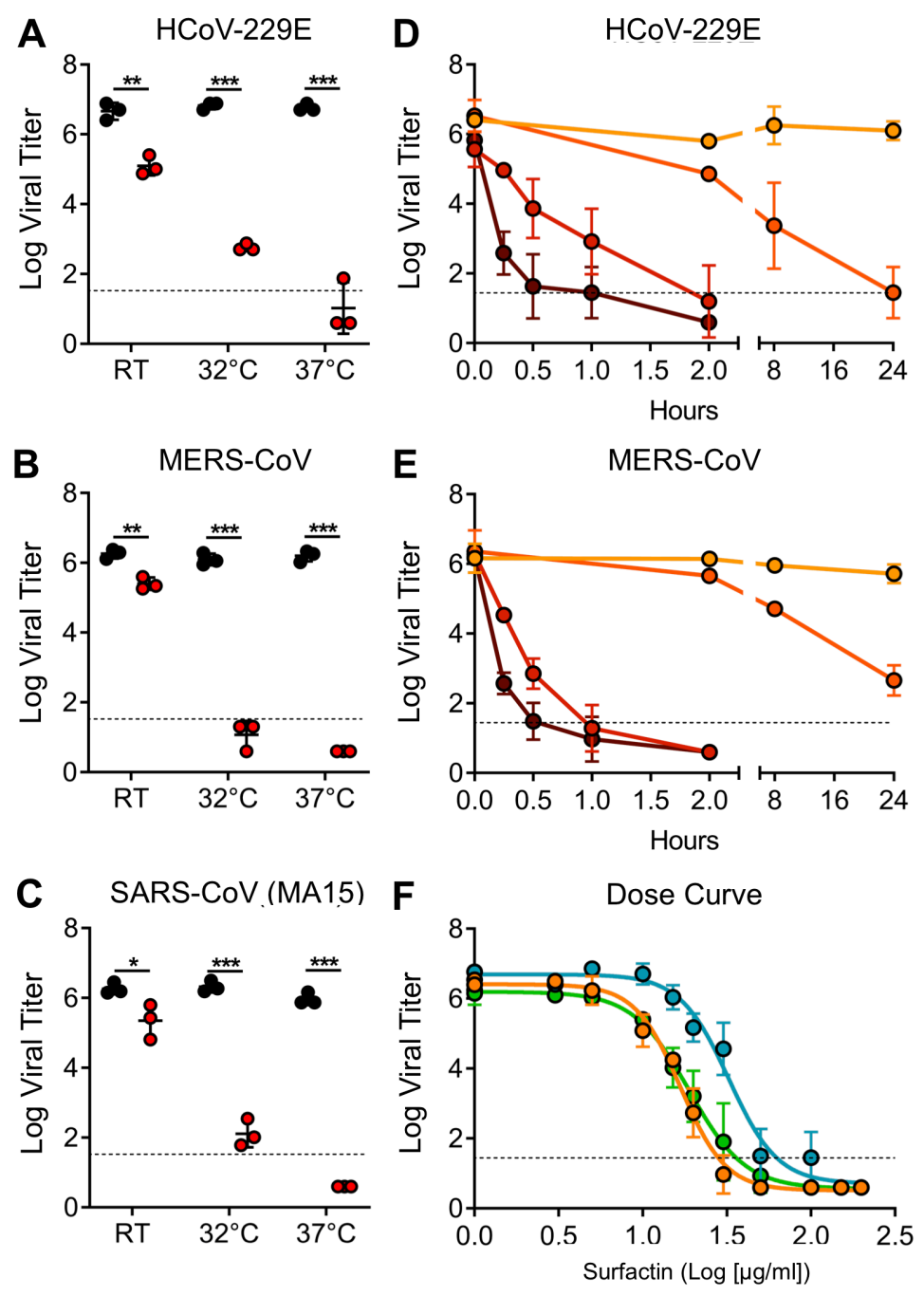

Figure 3: Characterization of CoV inhibition by surfactin. (A) HCoV-229E, (B) MERS-CoV, and (C) SARS-CoV (MA15) were treated PBS alone (black) or surfactin (red) and at room temperature (RT), $32^{\circ} \mathrm{C}$, and $37^{\circ} \mathrm{C}$ and infectivity determined $(n=3)$. HCoV-229E (D) and MERS-CoV (E) were treated for the indicated time at $4^{\circ} \mathrm{C}$ (light orange), RT (dark orange), $32^{\circ} \mathrm{C}$ (light red), or $37^{\circ} \mathrm{C}$ (dark red) and infectivity determined $(n=3)$. (F) HCoV-229E (blue), MERS-CoV (orange), and SARS-CoV MA15 (green) were diluted over a range of concentrations of surfactin and viral infectivity determined $(n=3)$. For dot plots, each point represents the titer from an independent experiment while the group mean is indicated by a line. Each point on the line graph represents the group mean. All error bars represent SD. The two tailed students t-test was used to determine P-values: ${ }^{*} P<0.05,{ }^{* *} P<0.01,{ }^{* * *} P<0.001$. 


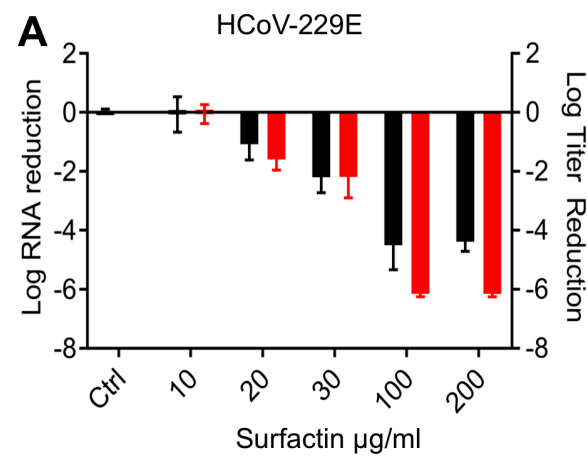

C
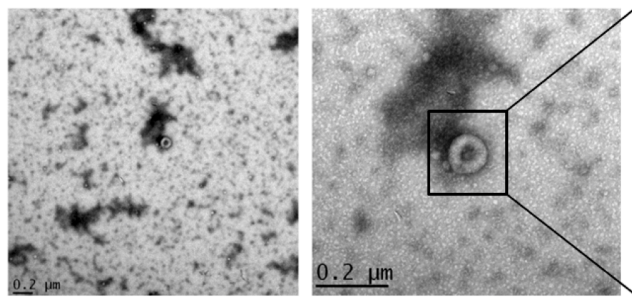

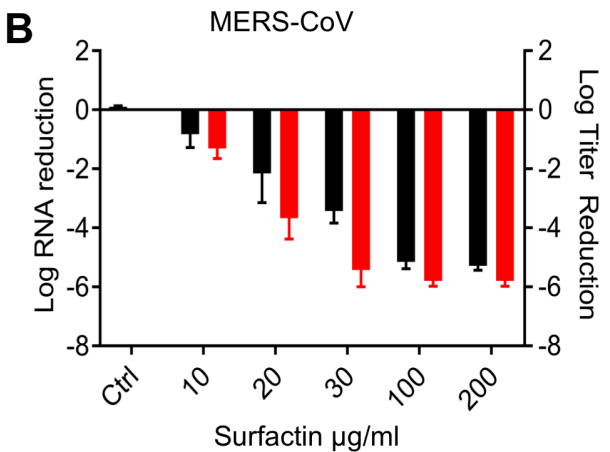

D
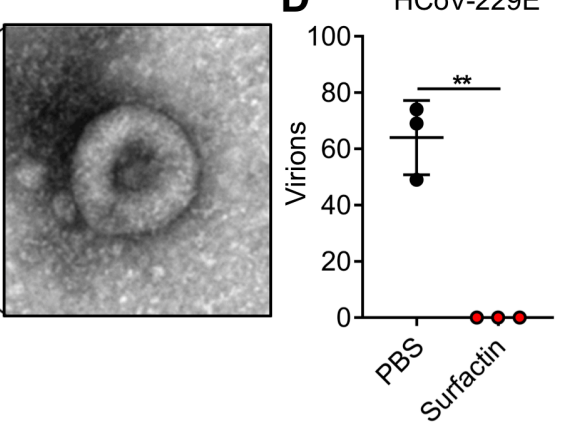

Figure 4: Surfactin disrupts CoV structural integrity. (A) HCoV-229E and (B) MERS-CoV were treated with the indicated concentrations of surfactin. Viral infectivity was then determined (red) or samples were then treated with RNAse I, RNA extracted, and viral genome copy number determined by RT-qPCR (black). (C and D) PBS or surfactin treated HCoV-229E samples negatively stained and examined by TEM and virions counted $(n=3)$. Representative micrograph shown in (C) while total counts are displayed in (D). Horizontal lines represent group mean while error bars represent SD. Two-tailed students t-test determined significance: ${ }^{*} \mathrm{P}<0.05,{ }^{* *} \mathrm{P}<0.01,{ }^{* * *} \mathrm{P}<0.001$. 

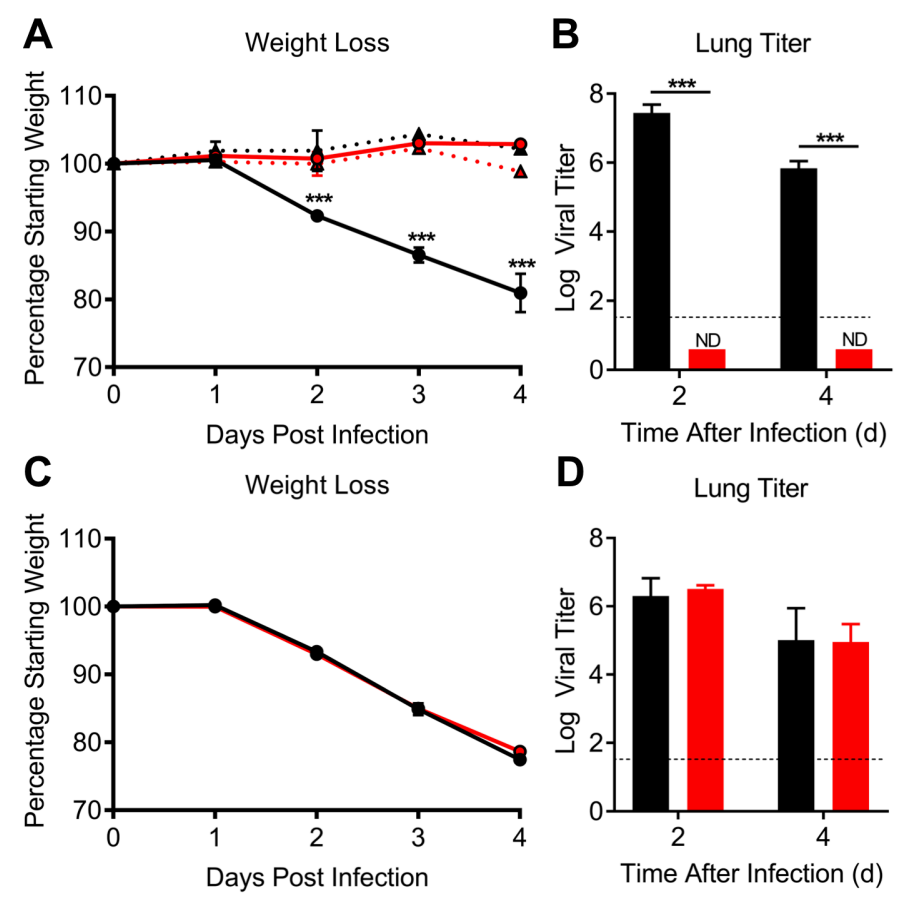

Figure 5: In vivo characterization of surfactin treatment on SARS-CoV infection. ( $A$ and $B$ ) BALB/C mice were then intranasally infected with $10^{4}$ PFU of PBS (black) or surfactin (red) treated SARS-CoV MA15 and (A) monitored for weight loss over 4 days. Dotted lines and triangles represent mock infected animals with PBS alone (black) or surfactin alone (red). (B) Lung tissue was harvested and viral titer determined at day 2 and day 4 . $n=4$ for all infected groups, $n=2$ for mock groups. ( $C$ and $D$ ) BALB/C mice were pretreated intranasally with $50 \mu \mathrm{l}$ of either PBS (black) or surfactin in PBS (red). 18 hours later, BALB/C mice were infected with $10^{4} \mathrm{PFU}$ of SARS-CoV (MA15) and (C) monitored for weight loss over 4 days. (D) Lung titer determined 2- $(n=5)$ and 4days post infection $(n=10)$. Dots on line graphs and bars on bar graphs represent the group mean. ND indicates that no titers were detected. All error bars represent SD. P-values were calculated using the two-tailed student's t-test, with: ${ }^{*} P<0.05,{ }^{* *} P<0.01,{ }^{* * *} P<0.001$. 

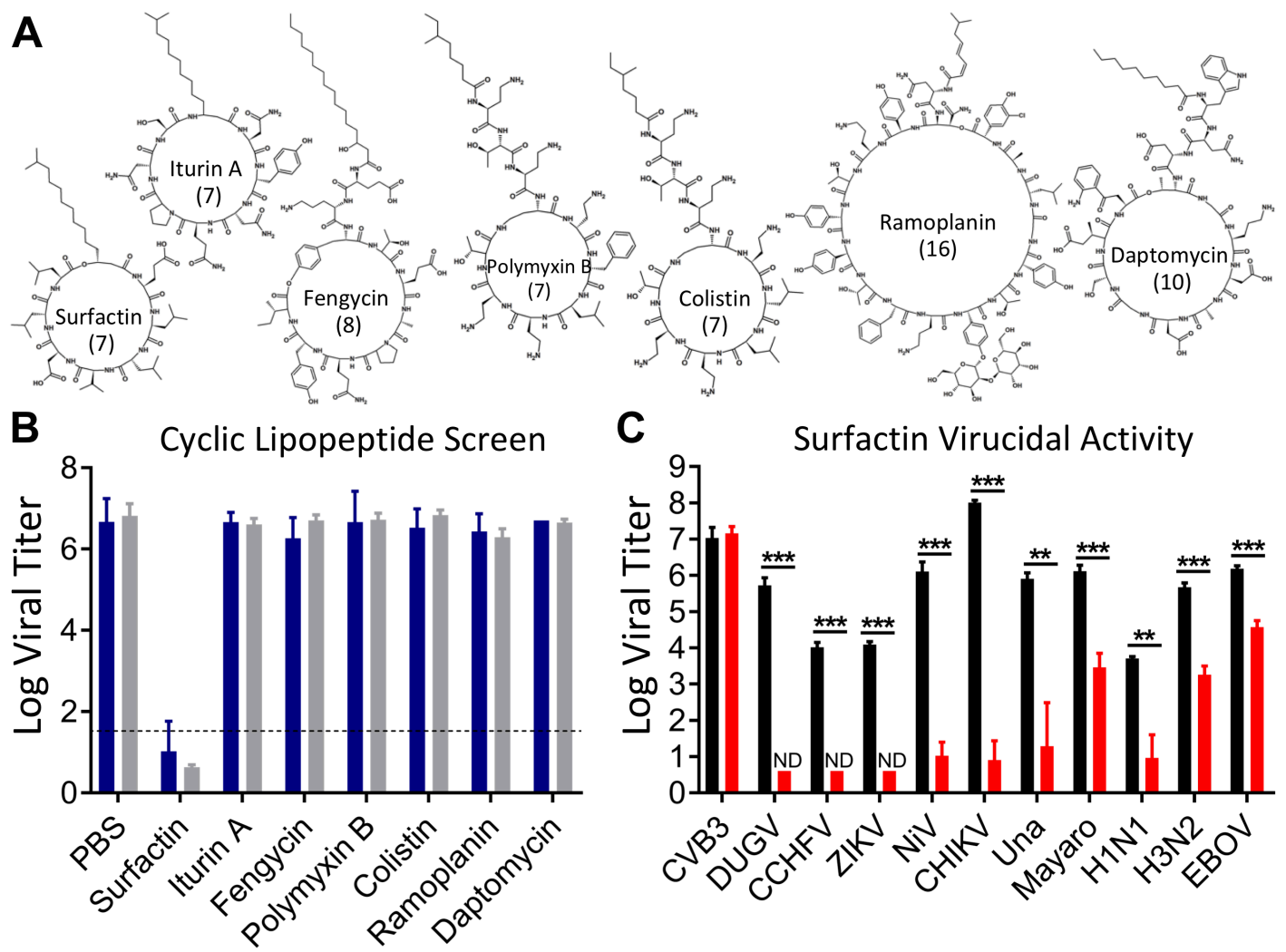

Figure 6: Surfactin, but not other cyclic lipopeptides, broadly reduce the infectivity of enveloped viruses. (A) Biochemical models of each of the seven cyclic lipopeptides tested. The number of amino acids present in the cyclic ring are shown in parentheses. (B) HCoV-229E (blue) and MERS-CoV (grey) were treated with PBS or the indicated cyclic lipopeptides in PBS and incubated for 2 hours at $37^{\circ} \mathrm{C}$. Viral infectivity was then determined $(n=3)$. (C) The indicated viruses were diluted PBS (black) or surfactin (red), incubated for 2 hours at $37^{\circ} \mathrm{C}$, and viral infectivity determined $(n=3)$. Viruses are abbreviated as follows: Coxsackievirus (CVB3), Dugbe (DUGV), Crimean-Congo hemorrhagic fever (CCHFV), Zika (ZIKV), Nipah (NiV), and Chikungunya (CHIKV), Una, Mayaro, Influenza A strains $\mathrm{H} 1 \mathrm{~N} 1$ and $\mathrm{H} 3 \mathrm{~N} 2$, and Ebola (EBOV). Bar graph bars represent the group means. Error bars represent SD. ND indicates that no titers were detected. The student's t-test was used to calculate $p$-values, with: * $P<$ $0.05,{ }^{* *} P<0.01,{ }^{* * *} P<0.001$. 